\title{
Course and Variations in Branches of Inferior Mesenteric Artery
}

\section{Date of Receiving: 5/01/2021}

Date of Review: 26/01/2021

Date of Acceptance: 11/02/2021

Prerna Neeraj Gupta ${ }^{1}$, Neeraj Gupta ${ }^{2}$

1. Assistant Professor, Department of Anatomy, Prathima Institute of Medical Sciences, Naganoor, Karimnagar, Telangana, India

2. Assistant Professor, Department of Forensic Medicine and Toxicology, Prathima Institute of Medical Sciences, Naganoor, Karimnagar, Telangana, India

DOI: 10.47799/pimr.0901.04

Address for correspondence: Dr. Neeraj Gupta, Assistant Professor, Department of Forensic medicine and Toxicology, Prathima Institute of Medical Sciences, Naganoor, Karimnagar, Telangana, India. Email: neeraj1982sep@gmail.com

\section{ABSTRACT:}

Background: The mesenteric blood supply is a combination of rich collateral networks. There are frequent anatomical variants encountered and these variations are sometimes involved in pathologies. Treatment of which requires a better understanding of the variations in the normal anatomy of the inferior mesenteric artery.

Methods: The present study was carried out in the Department of Anatomy, Prathima Institute of Medical Sciences, Karimnagar. A total of $n=50$ specimens, with $n=17$ adult males and $n=2$ adult female cadavers and fetuses of which $n=26$ were term and $n=2$, was preterm. Female fetuses $n=2$ of term and $n=1$ preterm were included in the study.

Results : The following variations were observed in the present study of course and variations in the branches of the inferior mesenteric artery and are grouped into three types.

Type I: In this type middle colic artery is arising from the inferior mesenteric artery instead of the superior mesenteric artery. It is a rare-variations and observed in a female fetus.

Type-II: Four Sigmoidal arteries are arising from an inferior mesenteric artery, after the origin of the left colic artery. This type is observed in a male fetus.

Type-III: Three Sigmoidal arteries are originated from the inferior mesenteric artery. This type was observed in a male adult and a male fetus.

Conclusion: Out of the 50 cases included in the study we found type 1 variation of IMA in $2 \%$ of cases, type 2 variation was found in $2 \%$ samples, and type 3 variation was found in $4 \%$ of samples. Based on the variations radiologists and Surgeons should be aware of possible consequences when doing colectomy, right hemicolectomy, left hemicolectomy, sigmoidectomy, en-bloc resection of the head of the pancreas, aneurysm, and chronic bowel ischemia. The present study is also useful for reconstructive surgeries in inferior mesenteric arteries in the case of ischemia.

Keywords: Inferior mesenteric artery, variations, branches INTRODUCTION :

The inferior mesenteric artery (IMA) is an unpaired visceral branch arising from the abdominal aorta $2-4 \mathrm{~cm}$, below the 3rd part of the duodenum the third main branch of the abdominal aorta, and normally arises at the level of L3. It transports and supplies arterial blood tothe derivatives of the hindgut, namely left $1 / 3 r d$ of the transverse colon, splenic or left colic flexure, and descending colon sigmoid colon rectum and the junction between upper $1 / 3$ rd and lower $2 / 3 r d$ of anacanal by anastomosis with the branches of other unpaired visceral branches of abdominal aorta -the branches of the superior mesenteric artery which is the artery supplying the derivatives of midgut i.e. lower half of 2 nd part of the duodenum, 3rd part and 4th part of the duodenum, caecum, vermiform appendix, ascending colon \& right $2 / 3$ rd of the transverse colon. ${ }^{[1]}$ The common branches of the inferior mesenteric artery are: 1) the left colic artery, which is located retroperitoneally and supplies the descending colon; 2) the sigmoid branches, whichsupply the sigmoid colon; and 3) the superior rectal artery or arteries, which are theterminal branches of the inferior mesenteric artery at the pelvic brim. However, the inferior mesenteric artery is known to show considerable anatomic variations. It has been reports of left colic artery arising from the abdominal aorta and the inferior mesenteric artery giving a branch to the left kidney or left renal artery arising from the inferior mesenteric artery and other variations. ${ }^{[2-5]}$ The pathological conditions where the Inferior mesenteric artery is involved are atherosclerosis, 
thromboembolism, aneurysm, and arteriovenous fistulas. Obstruction or constriction of the inferior mesenteric artery may be the cause of ischemia of the distal part of the gut.Knowledge of anatomical variations of IMA is required for planning radiological and surgical procedures of the distal third of the transverse colon, splenic flexure, descending colon, sigmoid colon, rectum, and deeply reaching interventions in the left iliac and hypogastric regions such as radical resection and preservation of left colic artery for rectal cancer or embolization. ${ }^{[6]}$ The current study aimed to determine the variations of the branch origin from the inferior mesenteric artery and its anatomical variations, including the occurrence of any additional arteries in our population.

\section{Material and Methods}

The present study was carried out in the Department of Anatomy, Prathima Institute of Medical Sciences, Karimnagar. Institutional Ethical committee permission was obtained for the study. Ethical protocol for obtaining the specimens for the study was followed.

Table 1: Showing the specimens included in the study

\begin{tabular}{|c|c|c|c|c|}
\hline \multirow{2}{*}{ Sex } & \multirow{2}{*}{ Adult } & \multicolumn{2}{|c|}{ Fetuses } & \multirow{2}{*}{ Grad Total } \\
\cline { 3 - 4 } & & Term & Pre-Term & \\
\hline Male & 17 & 26 & 02 & 45 \\
\hline Female & 02 & 02 & 01 & 05 \\
\hline Total & 19 & 28 & 03 & 50 \\
\hline
\end{tabular}

All the fetuses were injected with 25 cc-100cc of $10 \%$ formalin into the cranial cavity and trunk (thorax and abdomen) depending on the life of the fetuses. All the adult bodies were preserved by standard embalming techniques. Fetuses, adult bodies were dissected to expose the Inferior Mesenteric Artery and its branches in the following manner, systematically.

a) A median incision extending from the xiphisternal joint to the superior margin of the pubic symphysis.

b) An upper transverse incision extending from the xiphisternal joint to the midaxillary line.

c) A lower transverse incision extending from the superior margin of public symphysis along the line of the inguinal ligament to the highest curvature of the lliac crest.
The incision is extended into all the layers of the anterior abdominal wall i.e., skin, superficial fascia (fatty and membranous layers), and Muscles. All the peritoneal connections of the liver to the diaphragm are cut. The lesser omentum is cut with all its contents.A pair of ligatures are tied at the abdominal part of the esophagus and another pair of ligatures at the pylorus of the stomach. The stomach was removed by cutting between the ligatures and peritoneal folds i.e.,the gastro-phrenic, the gastro-splenic ligaments, and anterior layers of the greater omentum. Later Inferior mesenteric artery is traced for its origin, branches, and variations.

\section{Results}

The following variations were observed in the present study of course and variations in the branches of the inferior mesenteric artery and those are grouped into three types.

Type I: In this type middle colic artery is arising from the inferior mesenteric artery instead of the superior mesenteric artery. It is a rare-variations and observed in a female fetus.

Type-II: Four Sigmoidal arteries are arising from an inferior mesenteric artery, after the origin of the left colic artery. This type is observed in a male fetus.

Type-III: Three Sigmoidal arteries are originated from the inferior mesenteric artery. This type was observed in a male adult and a male fetus.

Level of Origin of inferior mesenteric artery: The inferior mesenteric artery is arising near the upper border in $n=9$ adult specimens and near the lower border in $n=10$ adult specimens. In the $n=31$ Fetuses inferior mesenteric artery arising near the upper border only. The details are entered in table No.1 and table No.2.

Table 2: Sex wise comparison of morphometric observations among fetuses

\begin{tabular}{|c|c|c|c|}
\hline Sex & Numbers & Mean Length & Std. Deviation \\
\hline Male & 22 & 28.73 & 4.621 \\
\hline Female & 9 & 28.89 & 3.919 \\
\hline Total & 31 & 28.77 & 4.364 \\
\hline
\end{tabular}

Table 3: Sex wise comparison of morphometric observation of inferior mesenteric artery among adults 


\begin{tabular}{|c|c|c|c|c|c|}
\hline \multicolumn{2}{|c|}{ Sex } & $\begin{array}{c}\text { Length } \\
\text { IMA }\end{array}$ & Length of LCA & Diameter of IMA & Diameter of LCA \\
\hline \multirow{3}{*}{ Male } & Mean & 5.776 & 9.86 & 5.94 & 3.76 \\
\cline { 2 - 6 } & Std. Deviation & 0.2386 & 1.383 & 1.391 & 1.033 \\
\hline \multirow{3}{*}{ Female } & Mean & 6.150 & 11.50 & 5.50 & 4.00 \\
\cline { 2 - 6 } & Std. Deviation & 0.3536 & 2.121 & 0.707 & 1.414 \\
\hline \multirow{2}{*}{ Total } & Mean & 5.816 & 10.03 & 5.89 & 3.79 \\
\cline { 2 - 6 } & Std. Deviation & 0.2672 & 1.490 & 1.329 & 1.032 \\
\hline
\end{tabular}

Table 4: Sex wise comparison of morphometric observations among fetuses

\begin{tabular}{|c|c|c|c|}
\hline Sex & Numbers & Mean Length & Std. Deviation \\
\hline Male & 22 & 28.73 & 4.621 \\
\hline Female & 9 & 28.89 & 3.919 \\
\hline Total & 31 & 28.77 & 4.364 \\
\hline
\end{tabular}

\section{Discussion}

In this current study, we found the inferior mesenteric artery is arising near the upper border in $n=9$ adult specimens and near the lower border in $n=10$ adult specimens. In the $n=31$ Fetuses inferior mesenteric artery arising near the upper border. The variation in the branches of the Inferior Mesenteric Artery noted in the present study are grouped into 3 types and compared with the observation of other studies.Type1:Middle colic artery which is normally a branch of SuperiorMesenteric Artery is arising from the Inferior Mesenteric Artery just above the origin of the left colic artery. Other sigmoidal arteries (2) are given from Inferior Mesenteric Artery and then it continues as a superior rectal artery, crosses left common iliac artery, and enters sigmoid mesocolon. This variation is seen only in one specimen out of fifty(2\%).Type 2 : in this variation, 4 sigmoidal arteries are given by IMA in the distal part in addition to the left colic arteries. sigmoidal arteries are anastomosing with each other by their branches and the proximal end of this anastomosis is joining with the descending branch of the left colic artery, the distal part of sigmoidal artery anastomosis is joining with the superior rectal artery branches.Type3: three sigmoidal arteries are given from the inferior mesenteric artery in two specimens. Type 2 and type 3 variations were observed by some authors. [7]Sunder land (1942)[8] reported that there were 1-7 sigmoid arteries from an inferior mesenteric artery in one of the specimens out of 25 dissections. Pillet J et al; [9]described inter mesenteric arcade which links the branches of SMAand IMA which explains the embryological persistence of the anterior anastomosis between the 13th, 21st, and 22nd primitive intestinal arteries. The name "Arc of Reolan" was given to the arterial arcade formed between a branch of the left colic and middle colic artery no other branches are given to the transverse colon from this arterial arcade. Loukas $\mathrm{M}$ et al; [10] presented a case report in which the right accessory renal artery arising as a common trunk with inferior mesenteric artery. In this case, other vascular variants were also noted. Left inferior phrenic artery arising from the celiac trunk, Left testicular artery arising from the left renal artery, and testicular vein emptying into renal veins. Yi SQ et al; showed a case of variations of the inferior mesenteric artery which was arising from the superior mesenteric artery. [11]

\section{Conclusion}

Out of the 50 cases included in the study, we found type 1 variation of IMA in $2 \%$ of cases, type 2 variation was found in $2 \%$ samples, and type 3 variation was found in $4 \%$ of samples. Based on the variations radiologists and Surgeons should be aware of possible consequences when doing colectomy, right hemicolectomy, left hemicolectomy, sigmoidectomy, en-bloc resection of the head of the pancreas, aneurysm, and chronic bowel ischemia. The present study is also useful for reconstructive surgeries in inferior mesenteric arteries in the case of ischemia.

\section{REFERENCES}

1. Moore KL, Dalley AF. Clinical Oriented Anatomy, 4th ed. Lippincott Williams\&Wilkins,Philadelphia 1999.

2. Chiene (1869) Complete Obliteration of the Cœliac and Mesenteric Arteries; the Viscera receiving theirSupply through the Extra-Peritoneal System of Vessels. Am J Med Sci 1869; 57:231-232.

3. Kostinovitch LI. A case of simultaneous occurrence of several variations of the visceralbranches of the abdominal aorta. Anat Rec 1937; 67:399-403.

4. Gwyn DG, Skilton JS. A rare variation of the inferior mesenteric artery in man. Anat Rec 1966;156:235-237.

5. Nuzhat A. Anatomy of Inferior Mesenteric Artery in Fetuses. Scientifica (Cairo) 2016:1-17. 
6. Samura M, Morikage N, Mizoguchi T, Takeuchi Y, Nagase T, Harada T, Suehiro K, Hamano KEffectiveness of Embolization of Inferior Mesenteric Artery to Prevent Type II Endoleak FollowingEndovascular Aneurysm Repair: A Review of the Literature. Ann Vasc Dis 2018; 11:259-264.

7. Balcerzak, Adrian Kwasniewska, OliwiaPodgórski, MichalOlewnik, LukaszPolguj, Michal. Types of inferior mesenteric artery: a proposal for a new classification. Folia morphologica. 2020;10.5603/FM.a2020.0115.vv.

8. Sunderland, S. Blood supply of the distal colon. The Australian and New Zealand Journal of Surgery 1942;11: 253-263.

9. Pillet J, Regnier B, Lhoste Ph, PilletJc, Mercier Ph, Cronier $P$ (1993) Considerations sur lavascularisation arterielle des colons. L'artèremésentériquemoyenne. Bull I'AssAnat 1993; 77(238): 27-30.

10. M Loukas, S Aparicio, A Beck, R Calderon, M Kennedy. A rare case of right accessory renal artery originating as a common trunk with the inferior mesenteric artery: $A$ case report. Clinical anatomy 2005; 18(7):530 - 55.

11. Yi Sq. Lij, Terayama H, Naito M, Kimura A. A rare case of inferior mesenteric artery arising from the superior mesenteric artery, with a review of the literature.Surg Radiol Anant 2008; 30(2):159-65.

How to cite this article : Gupta P, Gupta N. Course and Variations in Branches of Inferior Mesenteric Artery. Perspectives in Medical Research 2021; 9 (1):17-20

DOI: $10.47799 /$ pimr.0901.04

Sources of Support: Nil, Conflict of interest: None declared 